\title{
DNA-binding sequence specificity of DUX4
}

\author{
Yu Zhang ${ }^{1,2}$, John K. Lee ${ }^{3}$, Erik A. Toso ${ }^{1,2}$, Joslynn S. Lee ${ }^{5}$, Si Ho Choi ${ }^{1,2,4}$, Matthew Slattery ${ }^{5}$, Hideki Aihara ${ }^{3}$ \\ and Michael Kyba ${ }^{1,2^{*}}$
}

\begin{abstract}
Background: Misexpression of the double homeodomain transcription factor DUX4 results in facioscapulohumeral muscular dystrophy (FSHD). A DNA-binding consensus with two tandem TAAT motifs based on chromatin IP peaks has been discovered; however, the consensus has multiple variations (flavors) of unknown relative activity. In addition, not all peaks have this consensus, and the Pitx1 promoter, the first DUX4 target sequence mooted, has a different TAAT-rich sequence. Furthermore, it is not known whether and to what extent deviations from the consensus affect DNA-binding affinity and transcriptional activation potential.

Results: Here, we take both unbiased and consensus sequence-driven approaches to determine the DNA-binding specificity of DUX4 and its tolerance to mismatches at each site within its consensus sequence. We discover that the best binding and the greatest transcriptional activation are observed when the two TAAT motifs are separated by a $C$ residue. The second TAAT motif in the consensus sequence is actually (T/C)AAT. We find that a $T$ is preferred here. DUX4 has no transcriptional activity on "half-sites", i.e., those bearing only a single TAAT motif. We further find that DUX4 does not bind to the TAATTA motif in the Pitx1 promoter, that PitX1 sequences have no competitive band shift activity, and that the Pitx 1 sequence is transcriptionally inactive, calling into question PITX1 as a DUX4 target gene. Finally, by multimerizing binding sites, we find that DUX4 transcriptional activation demonstrates tremendous synergy and that at low DNA concentrations, at least two motifs are necessary to detect a transcriptional response.
\end{abstract}

Conclusions: These studies illuminate the DNA-binding sequence preferences of DUX4.

Keywords: DUX4, Facioscapulohumeral muscular dystrophy, FSHD, SELEX

\section{Background}

Facioscapulohumeral muscular dystrophy (FSHD) is arguably the most prevalent genetic disease of muscle $[1,2]$. It is caused by altered regulation of the subtelomeric chr4q macrosatellite repeat, D4Z4 [3-6]. This 3.3-kb macrosatellite sequence is typically present in $\sim 30$ tandem copies [7], while most cases of FSHD involve array contractions bringing the number of tandem repeats down to 10 or fewer $[8,9]$. When this occurs, the array, which is normally silent through a poorly understood repeatinduced silencing mechanism, becomes transcriptionally active $[10,11]$. Alternatively, the array can become transcriptionally active through second site mutations in genes required for repeat-induced silencing, for example,

\footnotetext{
* Correspondence: kyba@umn.edu

'Lillehei Heart Institute, University of Minnesota, Minneapolis, MN 55455, USA ${ }^{2}$ Department of Pediatrics, University of Minnesota, Minneapolis, MN 55455, USA

Full list of author information is available at the end of the article
}

SMCHD1 [12-14]. When this happens in the context of an allele that provides a downstream polyA signal [15], a muscle pathology ensues.

The D4Z4 repeat contains an open reading frame encoding DUX4, a double-homeodomain transcription factor [16]. The DUX4 protein is quite difficult to detect in FSHD clinical specimens $[17,18]$, but its presence can be read out indirectly in both proliferating and differentiating myoblasts, with slightly greater expression in the latter $[19,20]$. When induced at low levels of expression in myogenic progenitor cells, DUX4 interferes with MyoD expression and impairs myogenic differentiation [21, 22]. High levels of DUX4 expression promote cell death [22, 23]. As the homeodomains of DUX4 fall within the paired homeodomain class, and indeed are very close in sequence to those of the skeletal muscle stem cell regulators Pax3 and Pax7 [24, 25], a model suggesting that skeletal myogenic phenotypes in FSHD may be due in part to competition with Pax3/7 for 
targets was proposed [22]. Supporting this possibility, overexpression of either Pax3 or Pax7, but not the more distantly related homeodomain protein HoxB4, significantly reduced the cytotoxicity of DUX4 [22]. The sequence binding preferences of both DUX4 and Pax3/7 have recently been described through interrogation of sequences falling under ChIP-seq peaks $[11,26]$. Although both sequences contain TAAT core motifs, they differ in that the DUX4 consensus site contains two TAAT motifs in tandem, while the Pax $3 / 7$ site contains the motifs in a head-to-head orientation (i.e., TAAT followed by ATTA). Besides binding sequences identified through ChIP-seq, DUX4 binding to a sequence in the promoter of Pitx 1 had been demonstrated by band shift assays [10,27]. The Pitx 1 sequence does not contain a tandem TAAT motif, but rather has two overlapping head-to-head motifs: TAATTA, and this motif is also present in the human PITX1 gene. Thus, from work to date, it is not entirely clear what sequences DUX4 can bind to. Specific tests comparing DUX4 activity on different flavors of target sequences have never been done.

Because of the central role that DUX4 plays in FSHD, an understanding of the DNA-binding activity of DUX4 is essential to a mechanistic understanding of the disease. We have taken unbiased and candidate-selected approaches to compare the DNA-binding and transcriptional enhancing activity of DUX4 on various known sequences as well as randomly generated variants. Using the DNA element of greatest potency, we also investigate the copy number dependency of transcriptional activation by DUX4.

\section{Methods}

\section{Reporter constructs}

The luciferase reporter construct pGL4-12X-DUX4 containing $12 \times$ DUX4 binding motifs (CT flavor: TAATCTAATCA) was synthesized by GENEWIZ (New Jersey) and subcloned into XhoI/HindIII linearized the pGL4Amp luciferase plasmid (Promega) using T4 ligation. To generate the $6 \times$ reporter, pGL4-6X-DUX4 6 motifs were removed from this construct using KpnI digestion, followed by T4 ligation. To generate the $24 \times$ construct, pGL4-24X-DUX4, we ligated an Xhol/SalI fragment from pGL4-12X-DUX4 into XhoI linearized pGL4-12XDUX4 plasmid and the correct orientation selected. All other luciferase plasmids were constructed by $\mathrm{T} 4$ ligation of XhoI/HindIII linearized pGL4-Amp(R) luciferase plasmid with corresponding PCR-amplified fragments using In-Fusion HD cloning (Clontech). PCR fragments and primer information are listed in Additional file 1: Table S1.

\section{Generation of DUX4-inducible 293T cells}

FUIGW-rtTA was constructed by inserting rtTA2(s)-m2 (amplified by PCR) into BamH1/EcoR1 FUIGW (Lyu et al.
2008). pSam2-iDUX4-Flag-UBC-puro, the doxycyclineinducible DUX4 lentivector, was generated in the following way: The polyA signal from SV40 was amplified from p2lox (Iacovino et al. 2011) and inserted into pSAM2 (Zhang et al. 2011) at the Not1 site. The Ubiquitin C promoter and EGFP from FUGW (Lois et al. 2002) was then inserted into Pac1/BsrG1-digested plasmid, replacing the sgTRE promoter. The puromycin resistance gene (PAC) was PCR amplified and used to replace GFP by in-fusion cloning (Clontech). DUX4 with a c-terminal Flag peptide was PCR amplified and inserted into EcoR1/Not1 digested plasmid to generate pSam2iDUX4-Flag-Ubc-Puro.

\section{Transfection and luciferase assays}

Prior to transient transfection, DUX4-inducible 293T cells were plated in 96-well dishes until cells reached $60 \%$ confluency. Each well of cells was transfected with 95 ng of pGL4 firefly luciferase reporter plasmid together with $5 \mathrm{ng}$ of Renilla luciferase control plasmid using TransIT-LT1 transfection reagent (Mirus Bio LLC). Doxycycline $(500 \mathrm{ng} / \mathrm{ml})$ was added into each well after 24-h post-transfection to induce DUX4 expression, and cells were lysed $48 \mathrm{~h}$ post-transfection for luciferase assays using the Dual-Glo Luciferase Assay System (Promega). For luciferase assays, $75 \mu \mathrm{l}$ of Dual-Glo luciferase assay reagent was added to each well and incubated at room temperature for $15 \mathrm{~min}$ before measuring the firefly luminescence. After measuring, $75 \mu \mathrm{l}$ of Dual-Glo Stop \& Glo reagent was added into each well and incubated at room temperature for $15 \mathrm{~min}$ to quench the firefly luciferase activity, after which Renilla luminescence activity was measured. Luminescence readouts were measured using Cytation 3 plate reader (Bio-Tek) under luminescence fiber mode with the Gain-value fixed at 135 . Firefly luminescence was first normalized to Renilla luminescence and scaled to fold of induction to the control well (no dox addition). Each reporter analysis was done in triplicate and repeated twice.

\section{Bacterial expression of the DUX4 $\mathrm{N}$-terminus}

The N-terminus of DUX4, containing the two homeodomains was expressed in bacteria using the plasmid pET28 with a tobacco etch virus nuclear inclusion A endopeptidase protease (TEV) site engineered between the His6 tag and protein. We altered the codon usage, PCR amplified from a synthetic construct (Genscript) using primers:

Dux4_TEV19G_NdeI:

CGGAATTCCATATGgaaaacctgtacttccagggtAGACGT CGCAGGTTAGTTTGGACAC and

Dux4_152Qstop_BamHI:

TCGCGGATCCTTACTGACCAGGGTGACGAGCACGTCTGTTTTG, 
and subcloned into the vector.

The DUX4-HD protein was produced in BL21(DE3) and purified by Ni-NTA IMAC affinity purification. The recombinant DUX4 protein (containing the $\mathrm{N}$-terminal His6 tag) was cleaved off with TEV protease, and the final protein was purified by size exclusion chromatography (Superdex 200). For later experiments, we used a His-tagged SUMO-fusion construct (pE-SUMO, LifeSensors) cloned with following primers:

DUX4_15Ser_BsmBI:

CCCCCTGCTAATCCGTCTCAAGGTtctAGAGGT

AGAGGTAGACGTCGCAGGTTAG

DUX4_155R_BsmBI/XbaI:

CCCCTGCTAATCCGTCTCTCTAGATTATCTACC ACCCTGACCAGGGTGACGAGC

\section{Band shift assays}

Band shift experiments were performed with double stranded oligos of the following sequences:

DUX4(TT) GGCAGTCTAATTTAATCAAGTCGGC

DUX4(CT) GGCAGTCTAATCTAATCAAGTCGGC

DUX4(TC) GGCAGTCTAATTCAATCAAGTCGGC

DUX4(CC) GGCAGTCTAATCCAATCAAGTCGGC

DUX4-del GGCAGTCTAATTAATCAAGTCGGC

DUX4-ins GGCAGTCTAATCTTAATCAAGTCGGC

MALR GGCAGTCTAATTGAATCAAGTCGGC

Pitx1-25 GGCAGTCTTCTAATTAGTAGTCGGC

Pitx1-30

CGGATGCTGTCTTCTAATTAGTTTGGACCC

SELEX2 GGCAGTCTAATTCAATCCAGTCGGC

SELEX3 GGCAGTCTAATTAGCTTTAGTCGGC

SELEX4 GGCAGTCTAATGTTTTATAGTCGGC

\section{Noncompetitive band shifts}

In a final volume of $30 \mu \mathrm{L}, 10 \mu \mathrm{L}$ of $100 \mu \mathrm{M}$ probe was mixed with $20 \mu \mathrm{L}$ of $250 \mu \mathrm{M}$ DUX4 HD protein (in $500 \mathrm{mM} \mathrm{NaCl}, 20 \mathrm{mM}$ Tris-Cl, pH 7.4). Samples were incubated on ice for $1 \mathrm{~h}$, then run immediately on a $3 \%$ agarose gel containing $0.5 \mu \mathrm{g} / \mathrm{ml} \mathrm{EtBr}$.

Competitive band shifts Ten microliters of $100 \mu \mathrm{M}$ probe was added to $16 \mu \mathrm{L}$ of milliQ $\mathrm{H}_{2} \mathrm{O} ; 2 \mu \mathrm{L}$ of DUX4 HD protein $(125 \mu \mathrm{M}$ in $50 \%$ glycerol, $250 \mathrm{mM} \mathrm{NaCl}$, $10 \mathrm{mM}$ Tris-Cl, pH 7.4) was then added, followed by $2 \mu \mathrm{L}$ of $100 \mu \mathrm{M}$ FAM-labelled CT probe (final volume of $30 \mu \mathrm{L})$. Samples were incubated on ice for $1 \mathrm{~h}$, then run immediately on a $3 \%$ agarose gel, with no EtBr.

\section{SELEX-seq: DNA pulldown assays}

We synthesized the following partially randomized single stranded oligonucleotides:

Synthetic Bait-1 target: TCGTCGGCAGCGTCAGATGTGTATAAGAGACAGNNNNNNNNTAATNNN NNNNNCTGTCTCTTATACACATCTCCGAGCCCAC
GAGAC (underlined sequences represent Nextera adapter sequences).

Synthetic Bait-2 target: TCGTCGGCAGCGTCAGATGTGTATAAGAGACAGNNNT'A'A'T'T'T'A'A'T'C'A' NNNCTGTCTCTTATACACATCTCCGAGCCCACGA GAC

$\mathrm{A}^{\prime}$ refers to a mixture of $91 \%$ dATP, and $3 \%$ each of the other deoxynucleotides and so on for T, C', and G'.

We generated double-stranded oligonucleotide from these using 15 cycles of amplification with the following primers: BaitF: TCGTCGGCAGCGTC, BaitR: GTCTCGTGGGCTCGG.

Prior to DNA pulldown, 250 ng of His-tagged DUX4 DNA-binding domain was incubated with $50 \mu \mathrm{l}$ of NiNTA resin (Thermo Scientific) in $500 \mu$ of NT2 buffer (20 mM Tris- $\mathrm{HCl}$ (pH 7.5), $100 \mathrm{mM} \mathrm{NaCl}, 0.05 \%$ Nonidet P-40) at $4{ }^{\circ} \mathrm{C}$ for $30 \mathrm{~min}$, followed by three washes using $500 \mu \mathrm{l}$ of NT2 buffer. Then, $3 \mu \mathrm{g}$ of bait DNA was incubated with resin-protein complex in $250 \mu \mathrm{l}$ of binding buffer (20 mM Tris- $\mathrm{HCl}(\mathrm{pH} 7.5), 100 \mathrm{mM} \mathrm{NaCl}$, $0.05 \%$ Nonidet P-40, $0.5 \mathrm{mM}$ EDTA, $100 \mu \mathrm{g} / \mathrm{ml}$ BSA, $35 \mu \mathrm{g}$ of poly $(\mathrm{dI}-\mathrm{dC})$ ) at room temperature for $15 \mathrm{~min}$, followed by six washes using $500 \mu \mathrm{l}$ of NT2 buffer [28].

Pulled down DNA was then eluted by boiling and amplified again for a second cycle of systematic evolution of ligands by exponential enrichment (SELEX). For bait1, we performed 5 cycles of pulldown; for Bait-2, we performed 3 cycles. For sequencing, the product was amplified for 10 cycles using different combinations of indexing primers

Bait-1 input: N501-N701; Bait-1 output: N501-N702; Bait-2 input: N501-N704; Bait-2 output: N501-N705. Forward indexing primer, N501: AATGATACGGCGACCACCGAGATCTACACTAGATCGCTCGTCGGCAGC GTC. Reverse indexing primers: N701: CAAGCAGAAGACGGCATACGAGATTCGCCTTAGTCTCGTGGGC TCGG; N702: CAAGCAGAAGACGGCATACGAGATC TAGTACGGTCTCGTGGGCTCGG; N704: CAAGCAGAAGACGGCATACGAGATGCTCAGGAGTCTCGT GGGCTCGG; N705: CAAGCAGAAGACGGCATACGAGATAGGAGTCCGTCTCGTGGGCTCGG

Fifty $2.5 \mathrm{M}$ base paired-end reads were then generated for each sample on an Illumina HiSeq Instrument.

\section{Analysis of SELEX-seq data}

The Bait-1 SELEX-seq data was analyzed using the SELEX Bioconductor package [29]. A fifth-order Markov model was constructed using control Bait-1 sequences (no DUX4 pulldown) to predict the number of 16-mer sequences in each initial library as described [30,31]. Sequence counts from the DUX4 pulldown of Bait-1 were compared expected counts as predicted using Markov model derived from control data to identify significantly enriched sequences. The top 20 enriched putative DUX4 
binding sequences each contained one of the four $11 \mathrm{bp}$ sequences described in Fig. 3.

\section{Results}

Measuring DUX4 transcriptional activation on identified target sequences

ChIP-seq analysis has identified a consensus containing two tandem TAAT motifs (TAAT[T/C][T/C]AATCA) [11]; however, the relative activity of DUX4 for the four individual motifs that match this consensus is unknown. We therefore began by transfecting luciferase reporters containing a single motif upstream of a minimal promoter on to $293 \mathrm{~T}$ cells that we modified for doxycycline-inducible DUX4 expression. We also tested the activity of half-sites, having only the second TAAT motif with the terminal CA. This analysis showed that although all four variants could be recognized by DUX4, resulting in dox-dependent luciferase induction, the motif containing a central cytosine followed by a thymidine (TAATCTAATCA) had the greatest transcriptional activity in vivo (Fig. 1a). We therefore used this as our baseline positive control for other experiments. Halfsites did not show luciferase induction, although duplicating the CAATCA half-site apparently created a binding site for an unknown DUX4-unrelated activator (seen by dox-independent high level of background).

We next tested the activity of other putative DUX4binding DNA sequences. The first sequence to which DUX4-binding was attributed was in the promoter of the murine Pitx1 gene [10]. Two publications have shown band shift activity in nuclear extracts of cells transfected with DUX4 expression vectors [10, 27]. The similarity of the DUX4 homeodomains to those of Pax3 and Pax7 [22] which recognize TAAT sequences suggested that DUX4 might recognize a TAAT sequence within the Pitx1 promoter. The sequence used in these

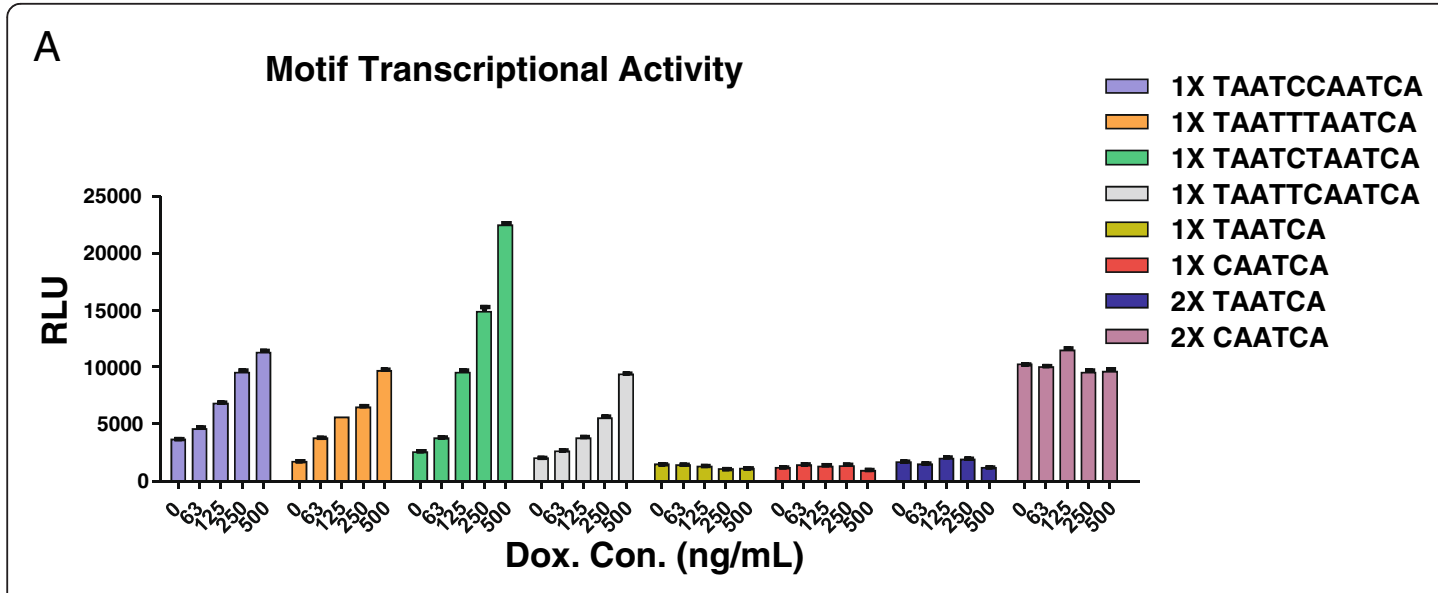

B

Motif Transcriptional Activity

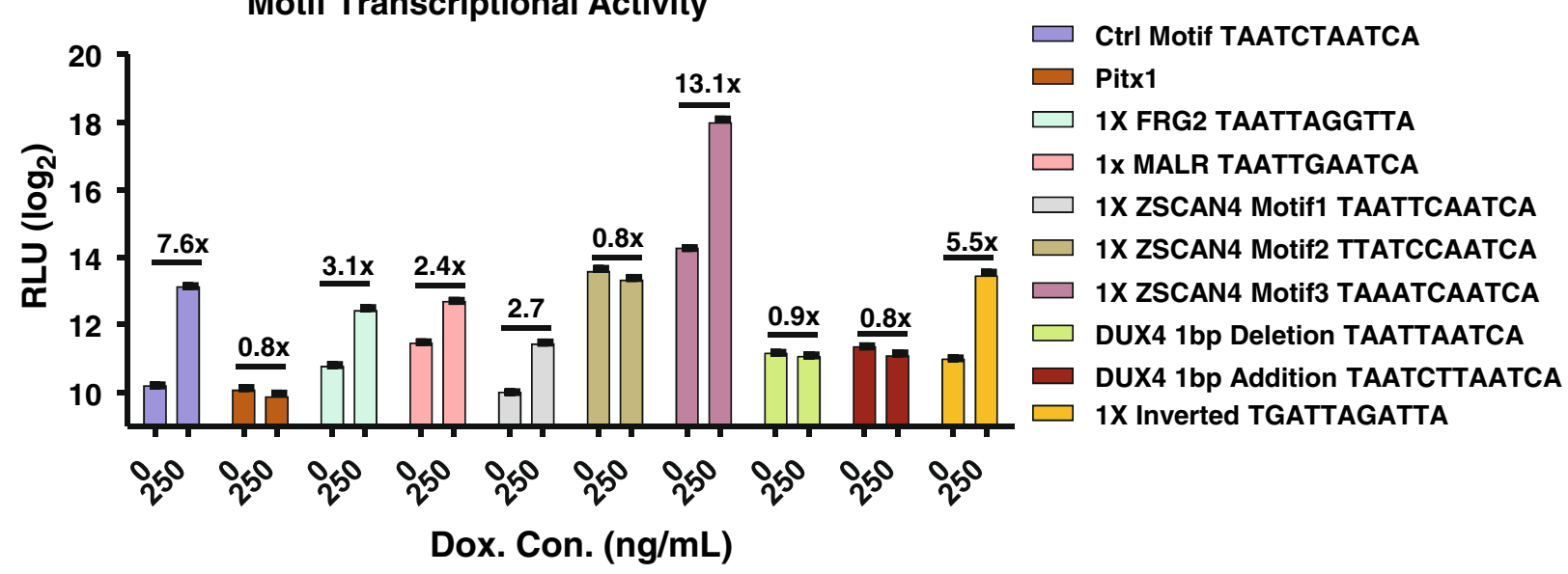

Fig. 1 DUX4-dependent luciferase activity of the four flavors of the DUX4 ChIP-seq motif. a DUX4 dose dependence of the four flavors of the DUX4 ChIP-seq consensus (left) and "half-sites" in one or two copies (right). RLU, relative luciferase units (normalized to Renilla luciferase). b DUX4-induced expression of luciferase reporters bearing various previously described DUX4 recognition motifs and mutations. Fold change is shown above each pair of assays. Note the log scale 
studies does contain a TAAT, but it is quite different from the ChIP-seq motif, described above. It is actually two overlapping TAATs on different strands (i.e., TAATTA); therefore, we generated a luciferase reporter containing one copy of the full 30-bp sequence used in these studies. It has also recently been shown that FRG2, a gene upregulated in FSHD, has motifs recognized by DUX4 [32]; we included the motif of greatest match to the consensus motif in our comparison: TAACCTAATTA. We also included a sequence from the MaLR repetitive element that is a near match to the consensus (TAATTGAATCA, note the middle $\mathrm{G}$ which deviates from the consensus) because a number of DUX4 targets have ChIP-seq peaks in nearby MaLR elements [11, 33]. Because of recent mobility of $M a L R$, a subset of $M a L R$ integrations are not shared between human and mouse, leading to concerns that a mouse model may not capture some relevant DUX4 target genes. One of the most strongly induced DUX4 target genes, ZSCAN4, has three potential DUX4 motifs. We included each of these motifs in our analysis. To complete this collection, we included two mutants in which the space between the two TAATs was increased or decreased by a single base, as well as the reverse complement of the control CT sequence to test the orientation dependence of the motif. The effect that background sequence may have on DUX4 binding is unknown, therefore, with the exception of the second and third ZSCAN4 motifs, all of these sequences were embedded into the background sequence flanking the first ZSCAN4 motif.

Cells were exposed to a relatively high dose of doxycycline (dox, $250 \mathrm{ng} / \mathrm{ml}$ ) and transfected with each reporter (Fig. 1b). In the presence of dox, the control TAATCTAATCA sequence gave about 8-fold higher expression over background, and the reverse complement gave about 6-fold increased expression, demonstrating orientation independence of transcriptional activation by DUX4. Other active sequences included those from FRG2 ( 3-fold), $\operatorname{MaLR}(2.4 \times)$, and motifs 1 and 3 from ZSCAN4 $(\sim 3 \times$ and $13 \times$, respectively) ZSCAN4 motif 2 , in which the $\underline{A}$ from the first TAAT was substituted, was inactive. The Pitx 1 sequence gave no activity. Neither did sequences in which the spacing between the two TAAT motifs was increased or decreased by a single base pair.

\section{Physical interaction between the DUX4 homeodomains and DNA}

The lack of induction by the 30-bp Pitx1 sequence was surprising, as it had previously been shown to interact with DUX4 in band shift assays. However, to date, published band shifts have been with nuclear extracts, not with purified protein. Therefore, given the striking lack of the consensus motif within the Pitx1 sequence, we hypothesized that the previously documented band shifts might not be the result of a direct interaction with DUX4. We therefore expressed the DNA-binding Nterminal domain of DUX4, which contains both homeodomains, in Escherichia coli and tested the ability of the DUX4 DNA-binding domain to directly interact with the various target sequences studied above (studies on full-length DUX4 were precluded by the insolubility of the bacterially purified full-length protein). We synthesized a series of double-stranded oligos, all of which contained the putative DUX4 interaction motif within the context of the same arbitrary identical $14 \mathrm{bp}$ of flanking sequence. Because the previously studied Pitx 1 sequence is $30 \mathrm{bp}$, we selected the central putative DUX4-interacting 13 nucleotides, containing the ATTAAT sequence, but we also tested the full 30-bp sequence independently. It is important to note that although the core ATTAAT sequence is conserved in the human PITX1 gene, there are several sequence differences outside of this core.

When this set of double-stranded oligos was simply mixed with excess protein, the band shift behavior of these sequences correlated roughly with their transcriptional activation potential: sequences that conferred DUX4-dependent luciferase induction also robustly shifted the probe, i.e., virtually all DNA was shifted, leaving no or almost no free probe (optimal "CT" motif and MALR, Fig. 2a). Note that the only molecules in this experiment are the protein and the DNA (visualized by ethidium bromide staining); therefore, the interaction is direct. The mutated DUX4 site, the half-site (i.e., TAATCA only) and the Pitx 1 core sequence in the context of the 25 mer oligo all showed no shift. On the other hand, the motifs with a single base insertion or deletion as well as the full 30-bp Pitx1 oligo showed some band shift activity, albeit with a significant unshifted band.

To more deeply investigate the relative affinity of DUX4 for these sequences, we performed competition experiments, in which we fluorescently labeled the "CT" consensus sequence (i.e., TAATCTAATCA) with FAM (carboxyfluorescein) and competed with the unlabeled sequences described above. For these experiments, the protein was limiting, thus even without competitor, only about $50 \%$ of the FAM-labeled probe was shifted. When unlabeled and added in excess, all four flavors of the DUX4 ChIP-seq motif competed equally well (Fig. 2b). However, when the other unlabeled sequences described above were used in excess, outside of the CT control, only the MaLR sequence displayed competitive activity, and it was less competitive than the CT control (Fig. 2c). Thus, while the insertion and deletion mutants and the Pitx1 30-bp oligo do have some ability to interact with DUX4 when the protein is in excess, when the protein is not in excess, these sequences cannot effectively compete for interaction with DUX4. 

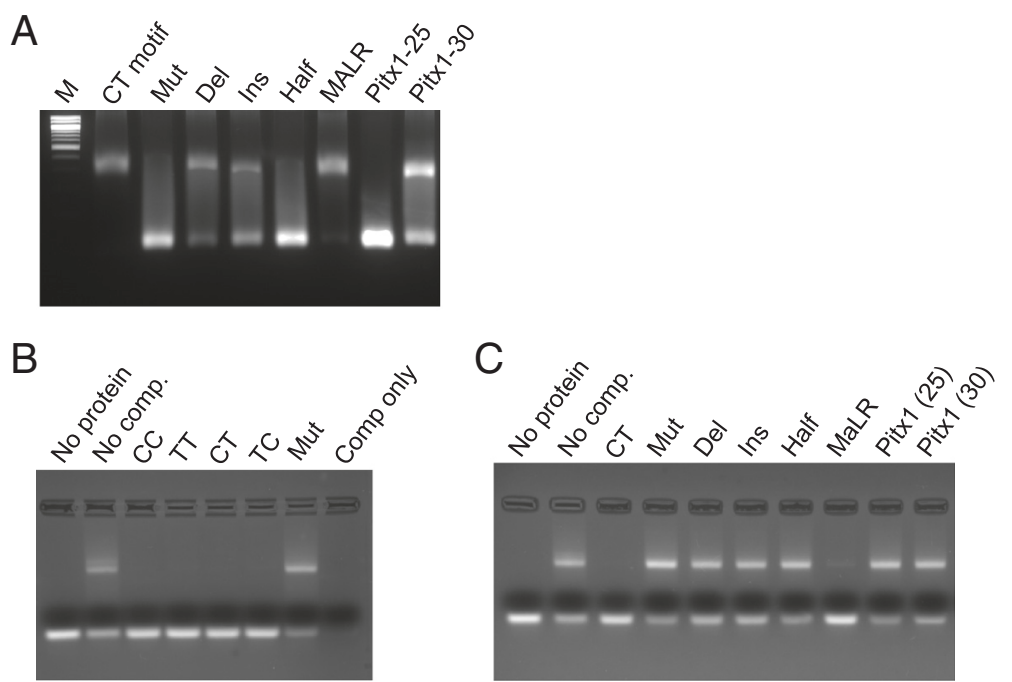

Fig. 2 Evaluating protein-DNA interaction by electrophoretic mobility shift. a Direct band shift assays for various sequence motifs. The DNA is detected by EtBr staining. The CT probe contains the control TAATCTAATCA sequence and shows a complete shift to the slower-migrating form in the presence of an excess quantity of the DUX4 DNA-binding domain. Other oligos showed various shift efficiencies. $\mathbf{b}$ Competitive band shifts of the four flavors of the DUX4 ChIP-seq consensus motif. The CT probe is labelled with FAM; thus, the gel has no EtBr, and binding is competed with unlabeled oligos. All four flavors of the DUX4 ChIP-seq consensus effectively compete away binding to the FAM-CT probe when provided in excess, while the mutant sequence fails to compete. c Competitive band shifts of the sequences tested for direct band shifting (in $\mathbf{a}$, above)

\section{Determination of DUX4-binding preferences by SELEX}

The experiments described above suggested that DUX4 interacts with variations on the double TAAT motif. To get a better sense of specific sequence preferences, we performed two SELEX-seq experiments. In the first experiment, we synthesized an oligo library based on the "TT" flavor of the ChIP-seq defined consensus: TAAT $\underline{\text { - }}$ TAATCA, and at each base, we introduced an error rate of $9 \%$ (i.e., $91 \%$ of the correct nucleotide, $3 \%$ of each incorrect nucleotide). We used the bacterially produced purified double homeodomain fragment to pull down this partially randomized oligo, and PCR amplified the pulled down products. In order not to completely lose lower-affinity sequences, we reiterated only to cycle 3 (Fig. 3a, Bait-2). A control, not pulled down sample, was also amplified three times to parallel the amplification to which the pulled down oligos were subjected. Following the third amplification, pulled down fragments were sequenced to a read depth of $2.5 \mathrm{M}$ and the frequency of each base at each position was compared in the pulled down vs. control library. At every position except for position 5 (the nucleotide between the two TAATs), the ratio was $>1$, meaning that the consensus sequence was selected over the possible mutants (Fig. 3b). At position 5 however, a strong preference for $\mathrm{C}$ rather than $\mathrm{T}$ was observed. Thus, the CT consensus, the most transcriptionally active of the four flavors initially tested (Fig. 1a), was also the most preferred sequence in this partially randomized library. Position 4 showed a detectable, but weaker, preference for $C$. This preference was not strong enough to result in selection against the consensus $\mathrm{T}$ at position 4 (the ratio for $\mathrm{T}$ was still just above 1 ), but the $\mathrm{C}$ was clearly positively selected, and preferred over A or G. In addition to preferences within the 11-bp defined motif, we identified preferences for sequence flanking the motif. Most obviously, G was selected against at all three upstream and downstream positions, while there was a moderate preference for $\mathrm{A}$ and $\mathrm{T}$ at these positions.

In the second SELEX-seq experiment, our objective was to determine whether there were other sequences, not involving the double TAAT motif, with which DUX4 could strongly interact. Based on the preference of individual paired class homeodomains for a TAAT sequence [34], we randomized sequence around a core TAAT motif. We performed pulldowns of these oligos, PCR amplified, and reiterated four times (Fig. 3a, Bait-1). After the fifth pulldown, DNA was sequenced, as above. We analyzed these sequences using a SELEX analysis Bioconductor package (see the "Methods" section) and identified enriched motifs. The top selected motif turned out again to be the CT consensus (TAATCTAATCA). The next two were similar to the ChIP-seq consensus, and the fourth was a sequence that did not resemble a double TAAT. We tested each of these sequences in luciferase reporter (Fig. 3c) and direct and competitive band shift (Fig. 3d, e) assays. The top two SELEX motifs showed transcriptional activation activity as well as strong direct and competitive band shift activity, with the CT consensus not surprisingly giving the greatest 
A

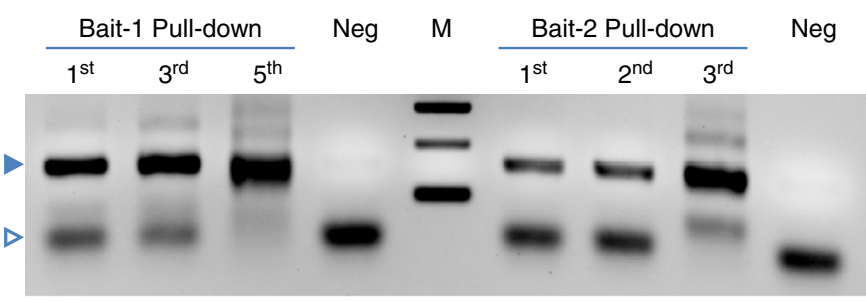

B

Ratio of Base Frequencies

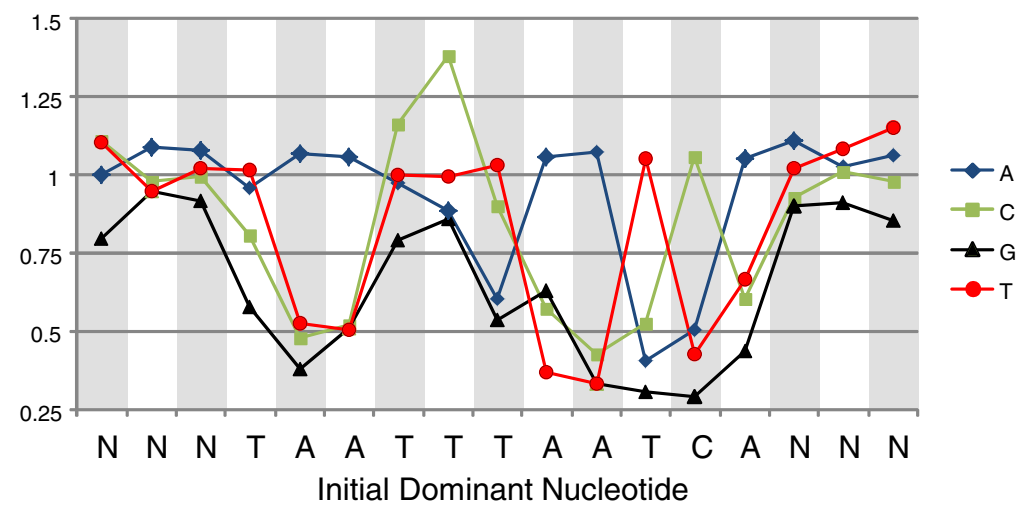

C

SELEX Motif Reporter Assay

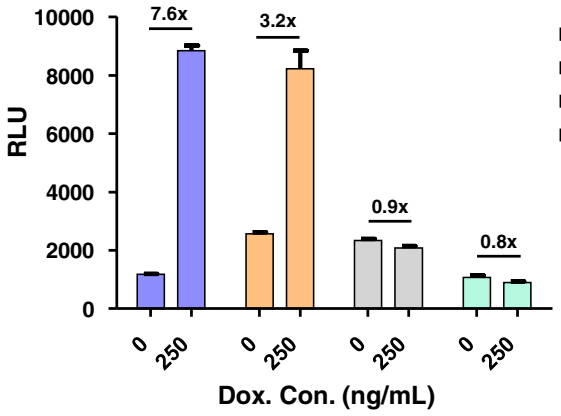

D

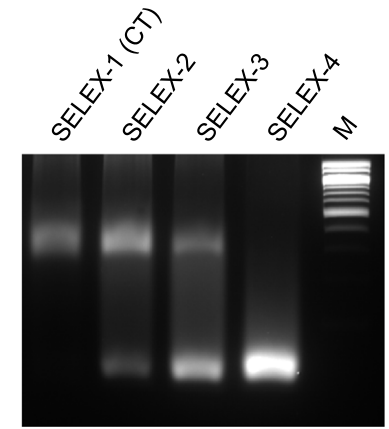

$$
\begin{aligned}
& \square \text { SELEX-1 (TAATCTAATCA = CT motif) } \\
& \square \text { SELEX-2 (TAATTCAATCC) } \\
& \square \text { SELEX-3 (TAATTAGCTTT = AAAGCTAATTA }) \\
& \square \text { SELEX-4 (TAATGTTTTAT) }
\end{aligned}
$$

E

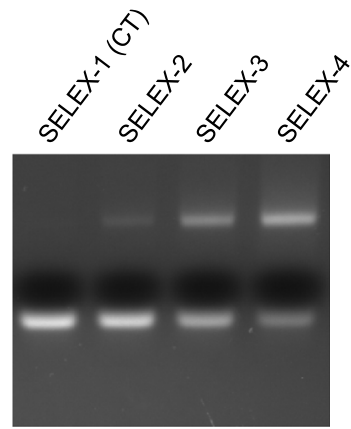

Fig. 3 Discovery of DUX4-interacting motifs by SELEX. a PCR assay after indicated rounds of DNA pulldown. Filled triangle indicates the specific band; open triangle indicates primer dimers. Note that the amplified product is enriched over primer dimers by the last cycle shown. $\mathbf{b}$ Selection bias for each base at each position (base frequency in the pulled down sequence/base frequency in the control). $\mathbf{c}$ Luciferase reporter assays for the top four SELEX-identified sequences. $\mathbf{d}$ Direct band shift assays for the top 4 SELEX motifs. e Competitive activity of the top four SELEX motifs. Each unlabeled SELEX probe was used to compete the shift of a FAM-labeled probe bearing the CT motif

activation and the best competition. The third motif, three mutations away from any ChIP-seq consensus, showed no transcriptional activity, weak band shift activity, and very weak competitive activity, while the fourth SELEX motif showed no activity in any assay. Thus, the second SELEX experiment did not find 
alternative motifs distinct from the double TAAT motif defined by ChIP-seq and furthermore independently identified the CT consensus as the most favorable of all randomly generated sequences.

\section{Synergy and spacing of clustered DUX4 binding sites}

With multiple independent lines of evidence pointing to the CT flavor of the DUX4 motif being the most preferred DNA-binding sequence, we next multimerized the CT motif and evaluated whether targeting multiple copies of DUX4 upstream of a minimal promoter would have additive or synergistic effects on transcription. This was motivated by the observation that many upregulated DUX4 target genes, for example, FRG2 and ZSCAN4, have multiple motifs to which DUX4 can bind [11, 32]. We generated reporters bearing $2,6,12$, or 24 copies of the CT motif and evaluated dox-dependent luciferase activity in DUX4-inducible 293T cells. With transfection conditions optimized for detecting activity of the single copy reporter, additional copies of the DUX4-binding sequence clearly had a synergistic effect, seen most clearly going from one copy to two copies, where two copies were $\sim 20$-fold more potent than a single copy at the highest concentration of dox (Fig. 4a). Adding additional copies increased expression, but this effect plateaued at six copies. However, when much lower amounts of DNA were transfected, which gave no detectable expression of the single copy reporter, more copies clearly made for a much more sensitive reporter, and this effect did not plateau up to 24 copies (Fig. 4b), indicating that the previous peak at 6 copies was due to the amount of protein being limiting for the amount of DNA used.

The nature of the cooperativity seen with two sites could mean that two DUX4 molecules bind cooperatively, or it could simply be due to increased avidity for a DUX4-interacting transcriptional coactivator. To investigate the first possibility, we generated a series of constructs in which the spacing between the two sites was varied (Fig. 4c). The previous multimer experiments inserted $10 \mathrm{bp}$, approximately one full helical turn, between each 11-bp motif, meaning that neighboring DUX4 proteins are binding on the same side of the DNA molecule. First, we placed two CT motifs directly adjacent to one another. This construct behaved like a single site, suggesting that DUX4 occupies a footprint greater than the $11 \mathrm{bp}$ that define its sequence specificity. We then generated a series of constructs with spacing increased in increments of half-turns: $15 \mathrm{bp}$, forcing the second DUX4 molecule to the opposite side of the DNA strand, and $20 \mathrm{bp}$, doubling the distance between the two DUX4 molecules. The transcriptional activation potential of all three constructs was roughly
A
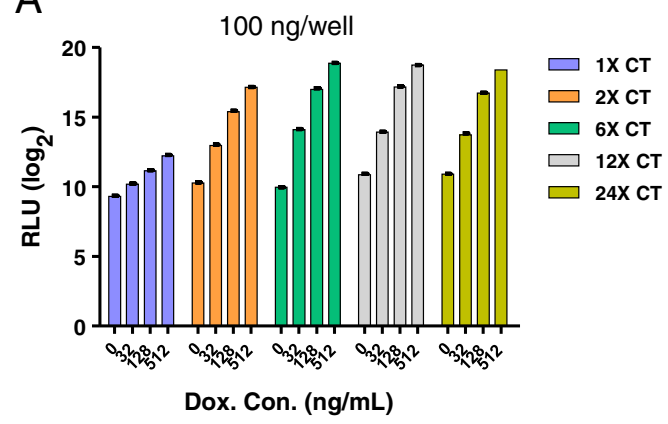

C

Spacing Synergy Effects

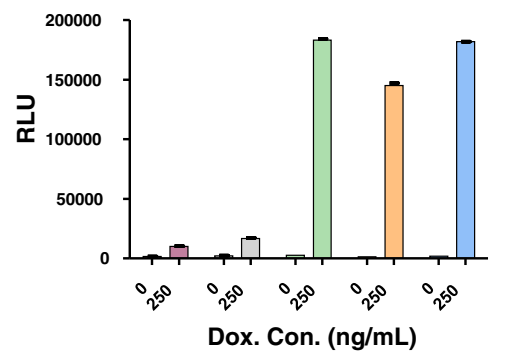

B

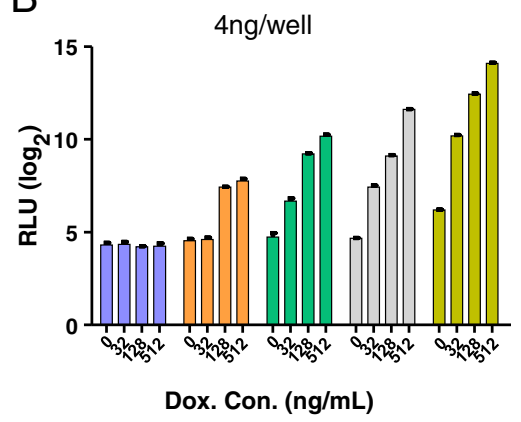

Fig. 4 Evaluating reporters with multiple DUX4 binding sequences. a Dose-dependent activity of reporters with 1-24 copies of the CT motif at $100 \mathrm{ng}$ of DNA/well. Note the logarithmic scale. $\mathbf{b}$ Dose-dependent activity of the same reporters at 4 ng/well. Synergy is demonstrated by the presence of activation with the $2 \times$ reporter but its absence with the $1 \times$ reporter at this concentration, as well as the continued increase in activity as copy number increases to $24 \times$. c Transcriptional activity of reporters with two CT motifs spaced at different distances (indicated), compared to a reporter with a single $\mathrm{CT}$ motif 
equivalent, suggesting that inter-molecular interactions of neighboring DUX4 molecules do not explain cooperativity.

\section{Discussion}

These studies have revealed a number of interesting properties of the DUX4 protein. First, multiple lines of evidence point to the CT motif, TAATCTAATCA, as the most preferred and most transcriptionally active of sequences that match the DUX4 consensus, previously defined by interrogation of sequences under DUX4 ChIP-seq peaks [11]. Both a direct comparison of all four flavors of the consensus sequence and two different random screens based on the pulldown of DNA sequences by the DUX4 DNA-binding domain showed the $\mathrm{CT}$ motif to be the most active.

Second, one of the sequences historically used to measure DUX4 activity, a DNA sequence from upstream of the mouse Pitx 1 promoter, has little activity in the assays used here. It is possible that various optimizations could bring out some weak activity, and indeed, the full 30-bp oligo used in previous studies had some modest band shift activity in our hands (although no competitive activity), but in direct comparisons with various motifs based on the double TAAT sequence, the Pitx1 sequence was inactive. Combined with the fact that this 30 -bp sequence is not completely conserved between mouse and human, that the expected ChIP-seq peak was not found over the region corresponding to this 30 -bp sequence in humans, or anywhere near PITX1 [11], and the fact that PITX1 was not found to be strongly upregulated by DUX4 in various human cell systems [11, 19] (or even mouse systems $[22,35,36])$, these data argue strongly against the model in which FSHD is caused by DUX4mediated overexpression of PITX1 [10].

Third, we find that single copies of DUX4 motifs are relatively ineffective at inducing transcription of target genes compared to double (or greater) copy numbers. This has implications for the relevance of mouse models for FSHD. It has been argued that the recent mobility of MaLR elements, which contain a single DUX4 motif, makes mouse a less than ideal model system to study DUX4 pathology, because the DUX4 binding sites created by a subset of these elements are in different places in the mouse genome compared to the human genome. Although the MaLR motif is a relatively strong individual motif, the fact that it is present only once within the $M a L R$ repeat means that most MaLR-associated DUX4 binding sites do not have the capacity for high level DUX4-mediated target gene expression. Although individual DUX4 transcriptionally responsive sites have been identified within primate-specific MaLR set [33], there is nevertheless a large and significant overlap between the sets of mouse and human DUX4-responsive genes [37].
These considerations argue that the mouse is not particularly disadvantaged as a model organism in which to study FSHD.

The paired class of homeodomain proteins typically bind DNA as dimers, in which each homeodomain interacts with a TAAT sequence [34]. The dimer is symmetrical over the DNA axis; therefore, the second homeodomain of the dimer binds over the reverse compliment, i.e., ATTA. Thus, paired class homeodomains recognize the palindromic TAAT- $\mathrm{N}_{X^{-}}$ATTA, and the orientation of the two homeodomains can be described as "head to head". The gap between the TAAT and ATTA may be two nucleotides (a "P2" site), as in the case of Pax7 [26], or three nucleotides (a P3 site), as in the case of Pax6 [31]. It is tempting to speculate that because the DUX4 homeodomains are physically connected by only a short linker peptide, this might force the homeodomains to bind DNA in a head-to-tail fashion, meaning that the DUX4 motif could be considered an N1 site, i.e., a non-palindromic TAAT pair with a single-gap nucleotide. On the other hand, the 11-bp recognition sequence for DUX4 is the correct size for a P3 sequence, albeit one with a mismatch in position 10 , rendering the ATTA into ATCA. Because the two homeodomains of DUX4 seem to have arisen from an internal duplication and are more similar to each other than to any other homeodomains, it seems improbable that they would recognize different core sequences. We await the structure of the DUX4-DNA complex to shed light on this question.

In the course of our study, we discovered certain sequences that have band shift activity when the protein is in excess, but failed to compete effectively when the protein is limiting and generally had no transcriptional activity. Because the DUX4 DNA-binding domain is actually two adjacent DNA-binding domains, we speculate that such band shifts may be due to DNA recognition by a single homeodomain only. Because half-sites did not have band shift activity, only certain TAAT sequences have this potential, most likely ones where the surrounding sequence tolerates and does not block positioning of the second homeodomain. This may open up an avenue for therapy development: if small molecules could be found that alter the interactions between the two homeodomains or between a homeodomain and DNA to stabilize such non-productive DNA binding, they might alter the DNA-binding specificity of DUX4 and thus diminish its toxicity.

\section{Conclusions}

These studies demonstrate that the optimal DNA sequence preferred by DUX4 is the $11 \mathrm{mer}$ TAATCTAATCA (the CT motif). Other than a weak band shift seen when protein is in molar excess, DUX4 does not 
interact physically or functionally with the Pitx 1 promoter sequence, but it does interact with numerous variants of the optimal CT motif. Although transcriptional activation by DUX4 on targets with a single DUX4-binding motif is relatively weak, DUX4 shows tremendous synergy when a two or more sites are present in the same target. This implies that animal species such as mice, with partially divergent MaLR repeats (which carry single motifs only), are not more particularly disadvantaged for reasons over and above their conventional genetic divergence from humans, with regard to their suitability for modeling the physiological effects of DUX4 expression.

\section{Additional file}

Additional file 1: Table S1. Oligos used in this study. (XLS $32.5 \mathrm{~KB}$ )

\section{Abbreviations}

ChIP: chromatin immunoprecipitation; FAM: carboxyfluorescein; FSHD: facioscapulohumeral muscular dystrophy; SELEX: systematic evolution of ligands by exponential enrichment.

\section{Competing interests}

The authors declare that they have no competing interests.

\section{Authors' contributions}

YZ and SHC designed and performed luciferase assays. YZ performed SELEX. $J K L$ and HA produced and purified the DUX4 protein and contributed to band shift assays. ET performed band shift assays. JSL and MS analyzed SELEX-seq data. MK conceived and designed the experiments and wrote the manuscript. All authors read and approved the final manuscript.

\section{Acknowledgements}

We thank Daryl Gohl and Aaron Becker for assistance developing the bait sequences and adapter-amplifying primers and Micah Gearhart for bioinformatics advice. This work was supported by the NIH (R01 AR055685) and by the Friends of FSH Research. We thank the Dr. Bob and Jean Smith Foundation for their generous support.

\section{Author details \\ 'Lillehei Heart Institute, University of Minnesota, Minneapolis, MN 55455, USA. ${ }^{2}$ Department of Pediatrics, University of Minnesota, Minneapolis, MN 55455, USA. ${ }^{3}$ Department of Biochemistry, Molecular Biology and Biophysics, University of Minnesota, Minneapolis, MN 55455, USA. ${ }^{4}$ Research Center, Dongnam Institute of Radiological \& Medical Sciences (DIRAMS), Busan, South Korea. ${ }^{5}$ Department of Biomedical Sciences, University of Minnesota Medical School, Duluth, MN 55812, USA.}

Received: 14 December 2015 Accepted: 8 January 2016 Published online: 28 January 2016

\section{References}

1. Orphanet. Prevalence of rare diseases: bibliographic data. Orphanet Report Series Rare Diseases collection 2010: www.orpha.net.

2. Deenen JC, Arnts H, van der Maarel SM, Padberg GW, Verschuuren JJ,

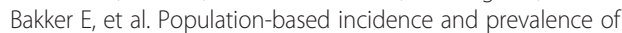
facioscapulohumeral dystrophy. Neurology. 2014;83:1056-9.

3. Gabellini D, Green MR, Tupler R. Inappropriate gene activation in FSHD: a repressor complex binds a chromosomal repeat deleted in dystrophic muscle. Cell. 2002;110:339-48.

4. Zeng W, de Greef JC, Chen YY, Chien R, Kong X, Gregson HC, et al. Specific loss of histone $\mathrm{H3}$ lysine 9 trimethylation and HP1gamma/cohesin binding at D4Z4 repeats is associated with facioscapulohumeral dystrophy (FSHD). PLoS Genet. 2009;5, e1000559.
5. van Overveld PG, Lemmers RJ, Sandkuijl LA, Enthoven L, Winokur ST, Bakels F, et al. Hypomethylation of D4Z4 in 4q-linked and non-4q-linked facioscapulohumeral muscular dystrophy. Nat Genet. 2003;35:315-7.

6. de Greef JC, Lemmers RJ, van Engelen BG, Sacconi S, Venance SL, Frants RR, et al. Common epigenetic changes of D4Z4 in contraction-dependent and contraction-independent FSHD. Hum Mutat. 2009;30:1449-59.

7. Schaap M, Lemmers RJ, Maassen R, van der Vliet PJ, Hoogerheide LF, van Dijk $H K$, et al. Genome-wide analysis of macrosatellite repeat copy number variation in worldwide populations: evidence for differences and commonalities in size distributions and size restrictions. BMC Genomics. 2013;14:143.

8. Wijmenga C, Hewitt JE, Sandkuij LA, Clark LN, Wright TJ, Dauwerse HG, et al. Chromosome 4q DNA rearrangements associated with facioscapulohumeral muscular dystrophy. Nat Genet. 1992;2:26-30.

9. van Deutekom JC, Wijmenga C, van Tienhoven EA, Gruter AM, Hewitt JE, Padberg GW, et al. FSHD associated DNA rearrangements are due to deletions of integral copies of a $3.2 \mathrm{~kb}$ tandemly repeated unit. Hum Mol Genet. 1993;2:2037-42.

10. Dixit M, Ansseau E, Tassin A, Winokur S, Shi R, Qian H, et al. DUX4, a candidate gene of facioscapulohumeral muscular dystrophy, encodes a transcriptional activator of PITX1. Proc Natl Acad Sci U S A. 2007;104:18157-62.

11. Geng LN, Yao Z, Snider L, Fong AP, Cech JN, Young JM, et al. DUX4 activates germline genes, retroelements, and immune mediators: implications for facioscapulohumeral dystrophy. Dev Cell. 2012;22:38-51.

12. Blewitt ME, Gendrel AV, Pang Z, Sparrow DB, Whitelaw N, Craig JM, et al. SmcHD1, containing a structural-maintenance-of-chromosomes hinge domain, has a critical role in X inactivation. Nat Genet. 2008;40:663-9.

13. Ashe A, Morgan DK, Whitelaw NC, Bruxner TJ, Vickaryous NK, Cox LL, et al. A genome-wide screen for modifiers of transgene variegation identifies genes with critical roles in development. Genome Biol. 2008;9:R182.

14. Lemmers RJ, Tawil R, Petek LM, Balog J, Block GJ, Santen GW, et al. Digenic inheritance of an SMCHD1 mutation and an FSHD-permissive D4Z4 allele causes facioscapulohumeral muscular dystrophy type 2. Nat Genet. 2012:44:1370-4

15. Lemmers RJ, van der Vliet PJ, Klooster R, Sacconi S, Camano P, Dauwerse JG, et al. A unifying genetic model for facioscapulohumeral muscular dystrophy. Science. 2010;329:1650-3.

16. Gabriels J, Beckers MC, Ding H, De Vriese A, Plaisance S, van der Maarel SM, et al. Nucleotide sequence of the partially deleted D4Z4 locus in a patient with FSHD identifies a putative gene within each 3.3 kb element. Gene. 1999;236:25-32

17. Snider L, Geng LN, Lemmers RJ, Kyba M, Ware CB, Nelson AM, et al. Facioscapulohumeral dystrophy: incomplete suppression of a retrotransposed gene. PLoS Genet. 2010;6, e1001181.

18. Jones TI, Chen JC, Rahimov F, Homma S, Arashiro P, Beermann ML, et al. Facioscapulohumeral muscular dystrophy family studies of DUX4 expression: evidence for disease modifiers and a quantitative model of pathogenesis. Hum Mol Genet. 2012;21:4419-30.

19. Rickard AM, Petek LM, Miller DG. Endogenous DUX4 expression in FSHD myotubes is sufficient to cause cell death and disrupts RNA splicing and cell migration pathways. Hum Mol Genet. 2015

20. Yao Z, Snider L, Balog J, Lemmers RJ, Van Der Maarel SM, Tawil R, et al. DUX4-induced gene expression is the major molecular signature in FSHD skeletal muscle. Hum Mol Genet. 2014

21. Dandapat A, Bosnakovski D, Hartweck LM, Arpke RW, Baltgalvis KA, Vang D, et al. Dominant lethal pathologies in male mice engineered to contain an X-linked DUX4 transgene. Cell Rep. 2014;8:1484-96.

22. Bosnakovski D, Xu Z, Gang EJ, Galindo CL, Liu M, Simsek T, et al. An isogenetic myoblast expression screen identifies DUX4-mediated FSHDassociated molecular pathologies. EMBO J. 2008;27:2766-79.

23. Kowaljow V, Marcowycz A, Ansseau E, Conde CB, Sauvage S, Matteotti C, et al. The DUX4 gene at the FSHD1A locus encodes a pro-apoptotic protein. Neuromuscul Disord. 2007;17:611-23.

24. Relaix F, Rocancourt D, Mansouri A, Buckingham M. A Pax3/Pax7-dependent population of skeletal muscle progenitor cells. Nature. 2005;435:948-53.

25. Seale P, Sabourin LA, Girgis-Gabardo A, Mansouri A, Gruss P, Rudnicki MA. Pax7 is required for the specification of myogenic satellite cells. Cell. 2000;102:777-86.

26. Soleimani VD, Punch VG, Kawabe Y, Jones AE, Palidwor GA, Porter CJ, et al. Transcriptional dominance of pax7 in adult myogenesis is due to highaffinity recognition of homeodomain motifs. Dev Cell. 2012;22:1208-20. 
27. Wallace LM, Garwick SE, Mei W, Belayew A, Coppee F, Ladner KJ, et al. DUX4, a candidate gene for facioscapulohumeral muscular dystrophy, causes p53-dependent myopathy in vivo. Ann Neurol. 2011;69:540-52.

28. Clouaire T, Roussigne M, Ecochard V, Mathe C, Amalric F, Girard JP. The THAP domain of THAP1 is a large $\mathrm{C} 2 \mathrm{CH}$ module with zinc-dependent sequence-specific DNA-binding activity. Proc Natl Acad Sci U S A. 2005;102:6907-12.

29. Rastogi C, Liu D, Bussemaker H. SELEX: functions for analyzing SELEX-sed data. R package version 1.2.0. 2015. http://bussemakerlab.org/software/ SELEX/.

30. Slattery M, Riley T, Liu P, Abe N, Gomez-Alcala P, Dror I, et al. Cofactor binding evokes latent differences in DNA binding specificity between Hox proteins. Cell. 2011;147:1270-82.

31. Czerny T, Busslinger M. DNA-binding and transactivation properties of Pax-6: three amino acids in the paired domain are responsible for the different sequence recognition of Pax-6 and BSAP (Pax-5). Mol Cell Biol. 1995:15:2858-71.

32. Thijssen PE, Balog J, Yao Z, Pham TP, Tawil R, Tapscott SJ, et al. DUX4 promotes transcription of FRG2 by directly activating its promoter in facioscapulohumeral muscular dystrophy. Skelet Muscle. 2014;4:19.

33. Young JM, Whiddon JL, Yao Z, Kasinathan B, Snider L, Geng LN, et al. DUX4 binding to retroelements creates promoters that are active in FSHD muscle and testis. PLoS Genet. 2013;9, e1003947.

34. Wilson D, Sheng G, Lecuit T, Dostatni N, Desplan C. Cooperative dimerization of paired class homeo domains on DNA. Genes Dev. 1993;7:2120-34.

35. Dandapat A, Hartweck LM, Bosnakovski D, Kyba M. Expression of the human FSHD-linked DUX4 gene induces neurogenesis during differentiation of murine embryonic stem cells. Stem Cells Dev. 2013;22:2440-8.

36. Krom YD, Dumonceaux J, Mamchaoui K, den Hamer B, Mariot V, Negroni E, et al. Generation of isogenic D4Z4 contracted and noncontracted immortal muscle cell clones from a mosaic patient: a cellular model for FSHD. Am J Pathol. 2012;181:1387-401.

37. Krom YD, Thijssen PE, Young JM, den Hamer B, Balog J, Yao Z, et al. Intrinsic epigenetic regulation of the D4Z4 macrosatellite repeat in a transgenic mouse model for FSHD. PLoS Genet. 2013;9, e1003415.

\section{Submit your next manuscript to BioMed Central and we will help you at every step:}

- We accept pre-submission inquiries

- Our selector tool helps you to find the most relevant journal

- We provide round the clock customer support

- Convenient online submission

- Thorough peer review

- Inclusion in PubMed and all major indexing services

- Maximum visibility for your research

Submit your manuscript at www.biomedcentral.com/submit 